\title{
Vergleich dreier Behandlungen der Lentigo maligna
}

\author{
Eine Lentigo maligna ist eine Präkan- \\ zerose, die entfernt werden muss. \\ Entscheiden sich Ärzte dabei für den \\ Laser statt fürs Skalpell, ersparen \\ sie ihren Patienten Schmerzen - bei \\ gleichem Erfolg.
}

- Wissenschaftler der University of Western Ontario in Kanada führten eine retrospektive Fallstudie mit 73 Lentigomaligna-Patienten zwischen 39 und 93 Lebensjahren (mittleres Lebensalter $=$ 64,8 Jahre) durch. Sie wurden zwischen Juli 1991 und Juni 2010 in Ontario behandelt.

27 Patienten wurden chirurgisch behandelt, 31 bestrahlt, 15 erhielten eine Laserbehandlung. Die mittleren Nachbeobachtungszeiten betrugen 16,6 Monate für die chirurgische Exzision, 46,3 Monate für die Strahlentherapie und 77,8 Monate für die Lasertherapie $(\mathrm{p}<$ $0,01)$. Die Rezidivraten lagen bei $4,2 \%$ für die chirurgische Exzision, 29\% für die Strahlentherapie und 6,7\% für die Laserbehandlung.

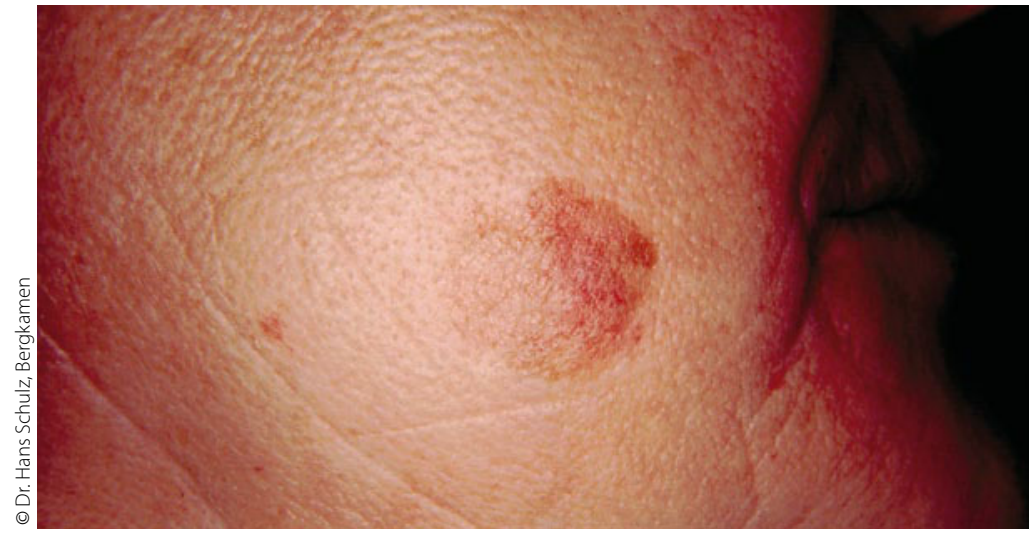

Lentigo maligna.

\section{Kommentar}

Als Goldstandard für die Behandlung von bösartigen oder potenziell bösartigen Hautveränderungen gilt der chirurgische Eingriff. Im Kopf- und Halsbereich ist aber das komplette Herausschneiden der Neoplasie nicht immer sinnvoll, meinen die Autoren. Der Nutzen einer Behandlung müsse immer im Zusammenhang mit den Folgen für den Patienten gesehen werden. So hätten nach einer operativen Entfernung viele Patienten mit großen Narben zu kämpfen, die durch den Laser vermieden werden können..

K. MALberg -

\footnotetext{
- H. Lee et al.

Carbon Dioxide Laser Treatment for Lentigo Maligna. A Retrospective Review Comparing 3 Different Treatment Modalities. Facial Plast. Surgery 13 (2011) 398-413
}

\section{Sport kann doppelt wirken}

\section{Sport hilft bei der Umstellung des Ernährungsstils. Einerseits erhöht er den Energieumsatz, andererseits verstärkt er den Hunger auf Gesundes. Doch das "Wie" ist noch wenig erforscht. Bei der Auswertung von zahlreichen, epidemio- logischen Studien entdeckten Wissenschaftler der Harvard University in Boston (USA) einen neuen gesundheitsfördernden Effekt von Bewegung.}

- Wer Sport treibt, ernährt sich gesünder. Regelmäßiges Training macht Appetit auf gesunde Nahrung und hilft, nicht zu viel zu essen. Sport fördert das Gespür für Sattheit. Gesunde Ernährung und sportliche Aktivität gehen Hand in Hand. Menschen, die anfangen, sich mehr zu bewegen, ernähren sich anschließend besser.

Die Arbeitsweise und Struktur des Gehirns wird durch Bewegung dauerhaft verändert, haben bereits frühere
Studien gezeigt. Regelmäßiger Sport verbessert die Gehirnleistung, erhöht die Menge der grauen Masse und präfrontalen Verknüpfungen, die für die die Ausführung von Handlungen und zielgerichtetes Verhalten zuständig sind. Die Neigung viel zu essen nimmt ab. Die Entscheidung für eine gesunde Ernährung fällt leichter.

\section{Kommentar}

In den beschriebenen Gehirnveränderungen könnte der Schlüssel liegen, den täglichen Versuchungen zu widerstehen, hochkalorisches Essen zu sich zu nehmen, so die Autoren. Bestehende Behandlungskonzepte gegen das Übergewicht könnten durch diese Wirkung des Sports verbessert werden.

K. MALBERG

- R. J Joseph et al.

(Korrespondenz: GL Blackburn, Beth Israel Deaconess Medical Center, Dept of Surgery, Center for the Study of Nutrition Medicine, Feldberg 880 , East Campus, 330 Brookline Ave, Boston, MA 02215, USA; e-mail: gblackbu@bidmc.harvard) The neurocognitive connection between physical activity and eating behaviour. Obesity Revs. 2011, 12: 800-812; doi: 10.1111/j.1467789X.2011.00893.x 